\title{
Erysipelothrix rhusiopathiae: bacteriology, epidemiology and clinical manifestations of an occupational pathogen
}

\author{
C. JOSEPHINE BROOKE* and THOMAS V. RILEY*† \\ * Department of Microbiology, University of Western Australia and †Division of Microbiology and Infectious \\ Diseases, Western Australian Centre for Pathology and Medical Research, Nedlands 6009 WA, Australia
}

\begin{abstract}
Erysipelothrix rhusiopathiae has been recognised as a cause of infection in animals and man since the late $1880 \mathrm{~s}$. It is the aetiological agent of swine erysipelas, and also causes economically important diseases in turkeys, chickens, ducks and emus, and other farmed animals such as sheep. The organism has the ability to persist for long periods in the environment and survive in marine locations. Infection in man is occupationally related, occurring principally as a result of contact with animals, their products or wastes. Human infection can take one of three forms: a mild cutaneous infection known as erysipeloid, a diffuse cutaneous form and a serious although rare systemic complication with septicaemia and endocarditis. While it has been suggested that the incidence of human infection could be declining because of technological advances in animal industries, infection still occurs in specific environments. Furthermore, infection by the organism may be under-diagnosed because of the resemblance it bears to other infections and the problems that may be encountered in isolation and identification. Diagnosis of erysipeloid can be difficult if not recognised clinically, as culture is lengthy and the organism resides deep in the skin. There have been recent advances in molecular approaches to diagnosis and in understanding of Erysipelothrix taxonomy and pathogenesis. Two PCR assays have been described for the diagnosis of swine erysipelas, one of which has been applied successfully to human samples. Treatment by oral and intramuscular penicillin is effective. However, containment and control procedures are far more effective ways to reduce infection in both man and animals.
\end{abstract}

\section{Introduction}

The genus Erysipelothrix consists of two named species, E. rhusiopathiae and E. tonsillarum, and an as yet unnamed third species. All are gram-positive, non-sporing rods. E. rhusiopathiae is a pathogen or a commensal or saprophyte of a wide variety of wild and domestic animals, birds and fish. Diseases of economic importance in animals include swine erysipelas, erysipelas of farmed turkeys, chickens and emus, and polyarthritis in sheep and lambs. Man can be infected; the organism is an occupational pathogen, prevalent in those working in association with animals and animal products. Three forms of human disease are recognised, the mildest and most common of these is the skin infection known as erysipeloid.

Received 19 Jan. 1999; accepted 15 March 1999.

Corresponding author: Professor T. V. Riley (e-mail: triley@cyllene.uwa.edu.au).
A review of E. rhusiopathiae and human infection has not been published for 10 years [1]. While few additional cases have been reported in this time, much has been achieved in the area of Erysipelothrix taxonomy and in molecular approaches to the diagnosis and pathogenesis of infection. It has been suggested that the frequency of human infection is declining, due to changes in technology by industry which have reduced the use of animal products and occupational exposure to the organism [1]. However, in specific environments, exposure to E. rhusiopathiae continues, and the threat of complications such as endocarditis is real. This review presents the recent developments against a background of older literature to ensure that awareness of the organism is maintained.

\section{Bacteriology}

History and nomenclature

Bergey's Manual [2] classifies E. rhusiopathiae as a regular non-sporing gram-positive rod. Until recently, 
the genus consisted of only the type species, $E$. rhusiopathiae, which was known to demonstrate considerable serological, biochemical and antigenic variation [3]. Genetic analyses have revealed a new species, E. tonsillarum [4]. Erysipelothrix was originally considered a close relative of the genus Listeria, although numerous molecular taxonomic studies have concluded now that the genus is a distinct cluster of organisms, most similar to the streptococci $[2,3]$.

E. rhusiopathiae (from the Greek 'erysipelas' - a disease, 'thrix' - a hair or thread, 'rhusius' - reddish and 'pathus' - disease [5]), literally 'erysipelas thread of red disease' [1], has a long history and is the result of many name changes. Koch [6] first isolated a strain of the genus Erysipelothrix in 1876 from a mouse he inoculated with putrefying blood. He described the organism as the 'bacillus of mouse septicaemia' and named it $E$. muriseptica. In 1882, Loeffler isolated a similar organism from cutaneous blood vessels of a pig which had died from swine erysipelas, and was the first to describe fully the infectious agent and the disease it caused in swine [6].

The first description of human disease, later attributed to Erysipelothrix, was reported in 1870 in the British Medical Journal; further cases were documented in 1873 as erythema serpens [7]. However, it was not until 1884 , when Rosenbach isolated an organism similar to Koch's from a patient with localised cutaneous lesions, that Erysipelothrix was established as a human pathogen. He coined the term 'erysipeloid' to differentiate between the human streptococcal disease erysipelas and the condition he had observed [6].

Rosenbach [6] distinguished three separate species of the organism, E. muriseptica, E. porci and E. erysiploides, based on their isolation from mouse, pig and man, respectively. It was later realised that these were three nearly identical strains of the same species [3], and they were named E. insidiosa, as originally proposed by Trevisan in 1885 . This name, and all 36 other documented names for the organism, were rejected in favour of E. rhusiopathiae in 1966, a combination which originated in 1918 [8].

Takahashi et al. [9] isolated a cluster of avirulent serotype 7 strains which were later found to be genetically distinct from $E$. rhusiopathiae by DNA base composition and DNA-DNA homology studies [4]. These strains formed the basis of a new species, $E$. tonsillarum (from the Latin 'of the tonsils'). Originally, E. tonsillarum was considered morphologically and biochemically identical to $E$. rhusiopathiae, but it was shown later that $E$. tonsillarum could ferment sucrose, while E. rhusiopathiae could not $[10,11]$. E. tonsillarum was described as avirulent for pigs, mice and chickens, but pathogenic for dogs $[12,13]$. There have been no studies so far into the pathogenicity of this species for man.

\section{Morphology and growth characteristics}

$E$. rhusiopathiae is a non-motile, non-sporulating, nonacid-fast, slender gram-positive rod, which is easily decolourised [2]. Gram-negative forms are often seen [14], particularly if the culture is old [15]; thus, the organism has been cited occasionally as a gramnegative bacillus [16]. E. rhusiopathiae was long described as non-capsulate [2], until recent studies showed the presence of a capsule and suggested a role for it in virulence [17].

Based on the colonial appearance of the organism, Erysipelothrix morphology is described as smooth (S) or rough $(R)[2,8,18]$. S-form colonies are convex, with a smooth surface and entire edge $[2,15,19]$. $R-$ form colonies are slightly larger with an irregular edge and a flattened, rough surface [14]. All colonies are clear, circular and very small $(0.1-0.5 \mathrm{~mm}$ diameter after $24 \mathrm{~h} ; 0.5-1.5 \mathrm{~mm}$ after $48 \mathrm{~h}[2,19])$, increasing in size and tending towards a pale blue opacity with further incubation or age $[2,19]$. Most strains exhibit a narrow zone of $\alpha$-haemolysis on blood agar, which can even show slight clearing after $48 \mathrm{~h}$ [2]. R-form colonies do not cause haemolysis [18].

Growth in broth was best described by Smith (cited by Jones [2]), who noted that the suspension had a 'faint opalescence ... which on shaking was resolved for a moment into delicate rolling clouds'. S forms cause slight turbidity and a powdery deposit, whereas $\mathrm{R}$ forms have a tangled hair-like appearance [3].

Cell morphology is closely linked to the colonial characteristics of each form [19]. S forms are slender, straight or slightly curved rods with rounded ends, $0.8-$ $2.5 \mu \mathrm{m}$ in length and $0.2-0.4 \mu \mathrm{m}$ in diameter. The rods exist in various formations, often as small chains [2]. The $\mathrm{R}$ form exhibits a predominantly filamentous morphology, frequently likened to the mycelial formations of fungi, although branching does not occur. The filaments can be $4 \mu \mathrm{m}$ to $>60 \mu \mathrm{m}$ in length and can have a beaded appearance with Gram's staining [2]. Long chains of distinct rods can also exist in this form $[14,18]$.

The origin of $\mathrm{R}$ and $\mathrm{S}$ forms and their role in disease have received much emphasis in the Erysipelothrix literature, possibly because different forms for other pathogens, such as Streptococcus pneumoniae, have distinct roles in virulence. For E. rhusiopathiae these roles are not definite, and there are conflicting observations on the role of each form. Furthermore, the distinction between $\mathrm{S}$ and $\mathrm{R}$ forms is not always clear. An intermediate (RS) form, which is the most common conformation [15], has been used to describe colonies sharing the characteristics of both types [8]. Some investigators have suggested that $\mathrm{S}$ forms are commonly isolated from acute pig diseases, such as septicaemia, and $\mathrm{R}$ forms from more chronic syn- 
dromes such as arthritis and endocarditis [8]. However, there are conflicting reports relating to the virulence of $\mathrm{R}$ forms. Gorby [20] reported that, in pig disease, $\mathrm{R}$ forms are the more virulent type, while Taylor [21] stated that the $\mathrm{R}$ form was generally considered less virulent. Similar possible relationships for $S$ and $R$ forms in human disease have not been documented.

Media and conditions of incubation (see below) play a major role in morphology formation; $\mathrm{R}$ forms are favoured by incubation at $37^{\circ} \mathrm{C}$ in acidic $\mathrm{pH}$, while $\mathrm{S}$ forms predominate in alkaline conditions ( $\mathrm{pH}$ 7.6-8.2) with incubation at $33^{\circ} \mathrm{C}$ [14]. Changing growth conditions has allowed $\mathrm{S}$ forms to give rise to $\mathrm{R}$ and $\mathrm{RS}$ forms, and $\mathrm{S}$ forms to originate from $\mathrm{R}$ forms [3]. The $\mathrm{S}$ form often dissociates to the $\mathrm{R}$ form with age $[8,15]$. These changes in morphology and cultural characteristics are reported to lead to changes in virulence and antigenic properties [8].

\section{Growth conditions and requirements}

E. rhusiopathiae is a facultative anaerobe [3]. Newly isolated strains are micro-aerophilic, but laboratoryadapted cultures grow both aerobically and anaerobically, with some strains being favoured by incubation in $\mathrm{CO}_{2} 5 \%$ or $10 \%$ [2]. The organism can grow at temperatures between $5^{\circ}$ and $44^{\circ} \mathrm{C}$ [2], optimally between $30^{\circ}$ and $37^{\circ} \mathrm{C}$ [18]. Best growth is favoured by an alkaline $\mathrm{pH}$. The optimum $\mathrm{pH}$ range has been documented as 7.2-7.6 $[2,5]$ or 7.4-7.8 [15, $18,19,22]$ and the limits of growth as 6.7-9.2 [23].

Growth is enhanced by the inclusion of serum $5-10 \%$, blood, glucose $0.1-0.5 \%$, protein hydrolysates, or surfactants such as Tween 80 in media $[8,18]$. The exact nutritional requirements of the organism are not known [18], but riboflavin, small amounts of oleic acid and several amino acids [24] - particularly tryptophan and arginine $[8,25]$ - are needed for growth. Higher concentrations of glucose and oleic acid are inhibitory [5].

\section{Biochemistry}

The genus Erysipelothrix is relatively inactive and gives negative results for catalase, oxidase, methyl red, indole and Voges-Proskauer reactions [15]. Carbohydrate fermentations produce acid without gas, but reaction patterns are variable and depend on the basal medium and indicator used $[14,26]$. Andrade's agar with horse serum $10 \%$ is the recommended medium for biochemical tests. The majority of strains produce $\mathrm{H}_{2} \mathrm{~S}$ gas, but again the extent of this production varies with the culture medium. The best reaction is demonstrated on triple sugar iron agar [27]. A more detailed description of the biochemical characteristics of Erysipelothrix can be found in the reviews of Ewald [8], Jones [2] or Reboli and Farrar [3]. Traditionally, biochemical reactions were used to differentiate be- tween Erysipelothrix and morphologically similar bacteria, such as Listeria and Corynebacterium spp. Characteristics used for this purpose included $\alpha$ haemolysis, lack of motility, lack of catalase production and resistance to neomycin [14]. While traditional biochemical testing may still be of value, particularly in discriminating between $E$. rhusiopathiae and $E$. tonsillarum, rapid identification can be achieved with an API Coryne System strip (bioMérieux) [28].

\section{Chemical tolerances}

E. rhusiopathiae is a remarkably resistant organism for one that does not form spores [18]. The survival of the organism in the environment is an important factor in the epidemiology of disease. E. rhusiopathiae is also tolerant to numerous chemicals. It can grow in the presence of phenol $0.2 \%$ and crystal violet $0.001 \%$, and is said to be one of the organisms most resistant to sodium azide, tolerating $0.1 \%[23,29]$. Some of these chemical tolerances have been utilised in the development of selective media.

\section{Antigenic structure}

Watts [30] noted that most strains of Erysipelothrix had two kinds of antigen, a species-specific heat-labile protein antigen and a heat- and acid-stable polysaccharide antigen, which now form the basis for serotyping strains. Dedié (cited by Reboli and Farrar [3]) recognised two major serotypes, A and B. Strains that did not react with these specific antisera were named group N. The Arabic numeral serotyping system of Kucsera [31] superseded the alphabetical scheme, due to variations in previous methods of antigenic extraction. The current standard serotyping method is a double agar-gel precipitation test with type-specific rabbit antisera and antigen prepared by hot aqueous extraction [31,32].

At present, strains of Erysipelothrix are classified as serotypes 1-26 [33]. A group $\mathrm{N}$ still exists for strains that have no type-specific antigen. In swine, $75-80 \%$ of isolates are of serotype 1 or 2 (previously group A or B) and the less common serotypes make up the remaining $20 \%$ [18]. Some investigators have noted a relationship between serotype and clinical condition in pigs, with serotype la most commonly isolated from acute swine illness, and serotype $2 \mathrm{a}$ more prevalent in chronic forms of disease [34]. However, other surveys have provided contradictory results, and all clinical conditions can be induced experimentally in susceptible swine with a variety of serotypes $[18,35]$. Sneath et al. [23] reported that strains from human or pig origin were antigenically similar, but there has been no verification of this since the numerical typing system was established. There is a deficiency in the literature regarding serotypes in human infection, and the epidemiological significance of serotyping in human disease is questionable. 
Molecular studies have cast further doubt on the value of serotyping as a reliable taxonomic and epidemiological method of classification. DNA-DNA hybridisation [10], polyacrylamide gel electrophoresis [36] and multilocus enzyme electrophoresis studies [11] classified serotypes 1a, 1b, 2, 4-9, 11, 12, 15-17, 19, 21 and 25 in the species E. rhusiopathiae, and serotypes $3,7,10,14,20,22-24$ in E. tonsillarum, with differing results for serotypes 13 and 18 [11]. When Erysipelothrix strains were analysed by restriction fragment length polymorphisms (RFLP), both $E$. tonsillarum and E. rhusiopathiae contained serotype 2 (virulent) and 7 (avirulent) strains. The creation of a third species of Erysipelothrix was suggested to account for a distinct cluster of strains [37].

These findings suggest that there is no direct relationship between serotype and virulence, supporting earlier investigators who noted that, within each serotype, strains of high, low and no virulence existed, and that factors other than serotype were important in the induction of disease in animal models $[38,39]$. Further investigation is required to reach a consensus on typing schemes and their role, if any, in studies of pathogenicity.

\section{Mechanisms of pathogenicity}

Relatively little is known about the pathogenesis of $E$. rhusiopathiae infections. Strains of E. rhusiopathiae are known to vary considerably in virulence. Despite much investigation, there has been no conclusive evidence of a relationship between virulence and morphology, chemical structure or antigenic structure [18]. Various virulence factors have been suggested, although their relative importance is not yet clear. The presence of a hyaluronidase and a neuraminidase has been recognised $[40,41]$, but hyaluronidase was detected in strains both virulent and avirulent for pigs. A correlation between the amount of neuraminidase produced and the virulence of strains was noted [41], although later studies demonstrated that avirulent acapsular mutant strains also produced the enzyme [17].

Adhesion to porcine kidney cells in vitro was greater for virulent strains [42]; however, the role of this factor in disease has not been investigated further. Further work on adhesion has been carried out [43], but this was in relation to arthritis in swine as a model for rheumatoid arthritis in man. Recently, the presence of a labile capsule was reported and acapsular mutants were constructed by transposon mutagenesis. In contrast to the parental strain, the mutants failed to resist phagocytosis by murine polymorphonuclear leucocytes, and could not survive within murine macrophages, suggesting that the capsule was an important virulence factor $[17,44]$. The mutant has been used in the development of a diagnostic PCR [45] (see below) and vaccine studies [46]. Further investigation of the pathogenesis of E. rhusiopathiae infection is required.

\section{Epidemiology}

E. rhusiopathiae and infections caused by this organism occur world-wide $[6,14]$. Infections of man and animals have been documented from Africa, Australia, several countries in the Americas, Japan, China and throughout Europe [8]. Human disease can originate from an animal or environmental source.

\section{Animal disease}

Swine erysipelas caused by E. rhusiopathiae is the disease of greatest prevalence and economic importance [18]. There are three clinical forms: a severe acute septicaemic form of sudden mortality; a milder, subacute urticarial form characterised by purple diamond-shaped lesions on the skin and a chronic form with endocarditis or arthritis [14]. Swine erysipelas is economically detrimental to the pig industries of North America, Europe, Asia and Australia [18].

As well as affecting swine, E. rhusiopathiae causes infections in a wide variety of domestic and wild mammals (including marine mammals), domestic, game and wild birds, and man [3, 8, 19, 22]. Polyarthritis of sheep and lambs, and erysipelas in calves, ducks and domestic turkeys are also economically significant diseases caused by $E$. rhusiopathiae [6, 19]. In Australia, the organism is an emerging problem in farmed emus [47].

Domestic swine are believed to be the most important animal reservoir of $E$. rhusiopathiae. The organism is shed by diseased animals in faeces, urine, saliva and nasal secretions, which can contaminate food, water, soil and bedding, leading to indirect transmission of the organism [18]. Furthermore, an average of $20-40 \%$ of healthy swine, and in some herds up to $98 \%$, harbour Erysipelothrix in the lymphoid tissue of the alimentary tract, particularly in the tonsils $[18,48,49]$. One study demonstrated that both virulent (serotypes 2, 6, 11, 12 and 16) and avirulent (serotype 7) serotypes were found on the tonsils [9]. Another showed that the faeces of apparently healthy animals contained virulent organisms [50]. The maintenance of E. rhusiopathiae in nature appears to result from asymptomatic carriage in animals and subsequent dissemination of the organism to the environment [6].

Mice are susceptible to infection [6], but other rodents seem to be affected only occasionally [51]. These animals can harbour the organism and are important reservoirs in some environments, such as meat packing plants [6]. Insects have been reported to carry $E$. rhusiopathiae, and are occasional vectors $[6,19,52]$; 
however, this is not a known route of infection for man.

\section{Environmental reservoirs}

The environment appears to be secondary in importance to animal reservoirs as a source of $E$. rhusiopathiae. However, in some circumstances, such as in marine environments, the organism may survive long enough to create a significant hazard to man [6].

E. rhusiopathiae is a saprophyte associated with some groups of animals, particularly marine fish, molluscs and crustaceans $[6,53,54]$. Freshwater fish and some species of bird are also hosts [14]. The organism survives and grows on the exterior mucoid slime of fish, without causing disease in the fish themselves [6]. It is likely that $E$. rhusiopathiae survives by a similar mechanism on the exterior slime layer of other marine creatures. The slime on fish appears to be an important source of infection for man. In early reports, fish caught under 'aseptic conditions' did not harbour Erysipelothrix [55], so investigators concluded that the organism was transmitted via the slime from other fish $[54,55]$. Boxes used for transport of fish seemed to play a vital role in the transmission of E. rhusiopathiae, and many human cases resulted from contact with these objects $[5,54,55]$.

Once in the environment, E. rhusiopathiae can survive for long periods although it does not form spores [18]. The organism is ubiquitous, and can be found wherever nitrogenous matter decomposes, retaining virulence and viability for months in putrid material [56]. Survival in swine faeces for 1-5 months, depending on seasonal conditions [19], and in soil for up to 5 years from the time of the last diseased pig [57], has been demonstrated. However, this latter report did not consider the possibility of asymptomatic shedding. E. rhusiopathiae has been recovered from sewage effluent from abattoirs, streams, drains and fertilizer $[3,15]$, surviving in drinking water for 4-5 days and sewage for 1014 days [5]. It was long thought that the organism could live in soil indefinitely, and early reports suggested that the source of infection was soil [5]. However, studies by Wood [58] did not support this widely held belief; E. rhusiopathiae survived for a maximum of only 35 days, depending on temperature and soil condition. Despite this limited endurance, the organism does survive long enough in soil to be a potential source of infection to animals and man.

E. rhusiopathiae persists in animal tissues for long periods, despite chilling, freezing, or curing [6]. The organism is resistant to pickling, smoking and salting [14]. Erysipelothrix can also survive in decaying tissue, and will remain viable in a carcass for 12 days in direct sunlight, for 4 months in putrefied flesh, for 9 months in a buried carcass and at least 10 months in refrigerated tissue [19]. E. rhusiopathiae has been isolated from fresh fish, pork and chicken for human consumption $[59,60]$. The widespread distribution of E. rhusiopathiae can be attributed to the ability of the organism to survive for long periods in the environment, and the fact that the organism can colonise or infect a wide variety of animals [6].

\section{Human infection}

Risk of humans infection is based on the opportunity for exposure, and factors such as age, sex, race and socio-economic status relate only to this opportunity $[3,61]$. Individuals involved in occupations or recreations with contact with animals, animal products or animal wastes are at greatest risk. Thus, E. rhusiopathiae infection is said to be occupationally related [56]. It follows that those in occupations with most frequent animal contact, such as butchers, abattoir workers, veterinarians, farmers, fishermen, fish-handlers and housewives are the most commonly infected $[5,6,19,62]$. However, cases have been documented from a very wide variety of occupations (see [1] for a complete list). The common names for human infection reflect this occupational mode of acquisition. These include seal finger, whale finger, blubber finger, fish hand, fish poisoning, fish handler's disease and pork finger $[3,6,62,63]$.

Human infection can occur from contact with infected animals, their secretions, wastes or products, or organic matter contaminated by any of these [6]. Infection is initiated either by an injury to the skin with infective material or when a previous injury is contaminated. There have been a few documented instances of penetration of the skin by the bacteria [61], and of infection by ingestion of contaminated food products [62]. There have been no reports of person-to-person transmission [3]. Modes of infection tend to be very occupation-specific, and transmission is generally by vehicles. These include contaminated objects causing wounds, such as knives, needles, dissecting instruments, fish teeth and spines, fish hooks, bone splinters, and crab, lobster and crayfish claws [6]. If a wound is already present, infection can result from contact with any of a very wide variety of contaminated objects $[6,62]$.

Infections in both man and animals appear to have a seasonal incidence, with most cases occurring in the summer months [6]. While it has been suggested that the biological activity of E. rhusiopathiae is related to temperature [55], others think that it is likely to be due to increased contact between people and sources of infection during these months [6].

\section{Clinical manifestations in man}

E. rhusiopathiae can cause three forms of human disease which closely resemble disease in swine. These 
are erysipeloid (a localised cutaneous form), a generalised cutaneous form and a septic form often associated with endocarditis [14]. It is possible that the incidence of human infection is declining. However, infection is possibly under-diagnosed, because of the resemblance it bears to other infections, difficulties in isolation and identification of the causative organism, and the rapid response to empiric antimicrobial therapy [64].

\section{Erysipeloid}

Erysipeloid is the most common form of human infection [14]. It is an acute localised cutaneous infection, described as a local cellulitis [3]. Erysipeloid usually occurs on the hand or fingers, reflecting the occupational nature of acquisition of the disease [62]; however, lesions have been described on many areas of the body [6].

The incubation period is usually $<4$ days, but can be up to 7 days after exposure [65]. The infection consists of a distinctive, well-demarcated, slightly elevated violaceous lesion [19]. The peripheral edge spreads slowly as the centre fades [66], and while vesicles are occasionally present, there is no suppuration or pitting $[14,56]$. There is associated local swelling, and an intense itching or a severe burning or throbbing pain [6], which is inconsistent with the mild look of the lesion [1]. Systemic symptoms can occur; $10 \%$ of cases experience fever and joint ache, and lymphadenitis and lymphadenopathy appear in $33 \%$ of patients [67]. Arthritis can manifest in an adjacent joint. The disease is self-limiting and usually resolves in 3-4 weeks without therapy [3], although relapses may occur if untreated [6].

\section{Diffuse cutaneous form}

This form is more generalised than erysipeloid, and includes the rare cases in which lesions progress from the initial site to other locations on the body, or in which there is development of lesions remote from the site of inoculation [68]. The lesions are similar to those of the localised form, but bullous lesions can also occur [69]. Systemic symptoms are more frequent and include fever, malaise, joint and muscle pain and severe headaches [6], and polyarthritis in rare instances. The clinical course is more protracted and recurrences are more frequent than with erysipeloid [56]. Very few cases have been documented; only 1 of 100 cases reported by Klauder [68] was generalised and none of the 500 cases reported by Nelson [67] was of this form.

\section{Septicaemia and endocarditis}

A more serious manifestation of E. rhusiopathiae infection is septicaemia, to which endocarditis has almost always been linked. In 49 cases of systemic infection in 15 years, $90 \%$ were associated with endocarditis [20]. Although septicaemia and endocarditis are relatively uncommon, there does appear to be an increase in incidence $[14,20]$, which could either reflect increased exposure or improved diagnosis.

E. rhusiopathiae endocarditis has a mortality of $38 \%$ and presents as an acute or subacute form, the latter being more frequent. In their summary of cases of endocarditis, Gorby and Peacock [20] discussed predisposing factors, and comparisons with other forms of endocarditis were made. E. rhusiopathiae endocarditis had an increased male to female ratio, possibly the result of occupational exposure, and mortality was almost double the rate of endocarditis of other aetiologies. The majority of patients had normal native heart valves and were immunocompetent. A history of alcohol abuse, believed to be a risk factor for the development of this complication, was noted in $33 \%$ of patients. Only $36 \%$ reported a previous erysipeloid lesion, and $89 \%$ of patients were in occupations involving contact with animals.

Recent reports $[16,70]$ have demonstrated that Erysipelothrix bacteraemia without endocarditis is more common than was thought previously, occurring mainly in immunocompromised patients. This increased rate was linked to a more thorough identification of blood culture isolates from these patients.

\section{Miscellaneous infections}

There are reports of other infections associated with Erysipelothrix. These have included chronic arthritis [69], cerebral infection, [71, 72] and osseous necrosis [68]. Recent case reports have focused on novel presentations and complications of Erysipelothrix infection. These have included erysipeloid with coexisting orf [64], persistent bacteraemia in a hospitalised patient [73], bacteraemia in an HIV-positive patient [74], endocarditis with acute renal failure [75], septicaemia and lupus nephritis [76], and septicaemia in a neonate [77]. In reports of systemic infection, the typical predisposing factors have been involved: either immunocompromised patients with atypical infection without cardiac involvement or immunocompetent patients with endocarditis. Renal involvement and alcoholism were factors noted in this second group.

E. rhusiopathiae has been implicated recently in a syndrome known as 'crayfish poisoning', which affects lobster fishermen in Western Australia and bears a clinical resemblance to erysipeloid [78]. A possible association between the organism and infections in fishermen was noted in 1947 by Sheard and Dicks [79], but proper identification was hampered by field conditions. It was not until 1996 that this infection was investigated further. The presence of other potential pathogens did not allow a direct causal relationship between $E$. rhusiopathiae and 'crayfish 
poisoning' to be established; however, the results were suggestive of this [78].

\section{Treatment and prevention}

Susceptibility data are still limited [3], despite recent reports on the subject $[9,80,81]$. Erysipelothrix is highly susceptible to penicillin, cephalosporins and clindamycin $[20,23,80]$. Most strains are resistant to aminoglycosides, trimethoprim-sulphamethoxazole, polymyxins, sulphonamides, streptomycin, novobiocin and vancomycin. The organism is variably susceptible to chloramphenicol, tetracyclines and erythromycin [3].

Erysipeloid can be treated effectively with oral penicillin [3]. Although infection is usually self-limiting, relapses and progression to more serious forms are possible. Oral penicillin will resolve a case of erysipeloid in around $48 \mathrm{~h}$, while intravenous penicillin is recommended for more serious E. rhusiopathiae infections [14]. While the mortality rate for endocarditis has been reduced from $100 \%$ in the pre-antibiotic era, there is still a $38 \%$ fatality rate despite available treatment [20]. This rate could be partly explained by the use of vancomycin, to which the organism is resistant, in empiric therapy for endocarditis [81]. Therefore, early diagnosis of all forms of E. rhusiopathiae infection is essential [14]. In those individuals allergic to penicillin, cephalosporins have been described as the most appropriate alternative, as clindamycin and erythromycin are only bacteriostatic towards E. rhusiopathiae [3].

Before the advent of penicillin therapy, some alleviation of symptoms could be achieved with hyperimmune serum. However, the resulting serum sickness was often more severe than an episode of erysipeloid, and this treatment was confirmed to be of little value for cutaneous infections [14, 82]. Commercial vaccines in the form of bacterins, lysates or live attenuated strains of E. rhusiopathiae serotype 2 offer protection to pigs and turkeys [25]. Vaccination is not a viable option in man, because clinical erysipeloid appears to convey little or no immunity $[55,66]$, as evidenced by relapse and/or re-infection.

Since Heilman and Herrel [52] first reported the success of penicillin therapy for Erysipelothrix infections, there has been no recorded resistance of the organism to this antibiotic. One experiment involving serial passage of 75 strains for 8 months did not produce resistance [14]. Plasmids were not found in $E$. rhusiopathiae in early investigations [80,81], but later studies were able to detect them [83]. Plasmids appear to play no critical role in E. rhusiopathiae resistance. Antibiotics contained in animal feed have been reported to influence the resistance of some strains of E. rhusiopathiae, although the mechanism remains uncertain [9].
Containment and control of E. rhusiopathiae are the most effective means of preventing the spread of infection in man and animals. An awareness of the infection is essential for individuals in occupations which put them at risk. Suggested preventive measures include: the wearing of gloves or other protective hand wear, good hygiene - especially frequent hand washing with disinfectant soap - and the prompt treatment of any small injuries [6]. Good health is considered an important factor in prevention, as a poor state of health, including alcoholism, may predispose to the serious forms of infection [20,84]. Control of animal disease by sound husbandry, herd management, good sanitation and immunisation is recommended [18].

The removal or regular disinfection of contaminated sources has been shown to be an important method of limiting the spread of the organism throughout a work environment [6]. E. rhusiopathiae can be killed by commonly available disinfectants [19]. However, many investigators have noted that structurally complex equipment is difficult to clean, and because the organism is able to survive in organic matter, disinfecting without cleaning is useless $[85,86]$. If disinfection is impractical, other control measures become even more significant.

Control of reservoir populations of E. rhusiopathiae is impractical or impossible, because of the widespread distribution of the organism, the large variety of animal hosts and its ability to persist in the environment. However, the possibility of human infection can be reduced by awareness, safe work practices and sensible precautions.

\section{Isolation and laboratory identification}

It has been reported that medical practitioners who see cases of human erysipeloid regularly find the lesion and other symptoms are 'so typical that a biopsy and subsequent isolation is neither necessary nor justifiable' [87]. As a result, the majority of identification protocols have been developed with swine erysipelas in mind. However, if human cases are declining, doctors could be less likely to recognise the infection, and, therefore, isolation techniques and methods for identification of $E$. rhusiopathiae may gain new importance.

\section{Cultural methods}

Traditional cultural methods for E. rhusiopathiae isolation involve the use of selective and enrichment media. Identification is based on Gram's stain, cultural morphology, motility, haemolytic characteristics and biochemical properties, particularly $\mathrm{H}_{2} \mathrm{~S}$ production [3]. For bacteraemia or endocarditis, a blood sample cultured in standard blood culture media is sufficient for isolation [19], as E. rhusiopathiae is not particu- 
larly fastidious [3]. The organism is more difficult to isolate from cases of erysipeloid, because it is said to live deep in the skin [2]. A biopsy at the advancing edge of the lesion and extending the entire thickness of the dermis is required. Aspirates of the lesion or associated bullae and vesicles are usually less rewarding [19]. Swabbing does not usually detect the pathogen [65].

Biopsies and aspirates are incubated in an infusion broth of glucose $1 \%$, in air or $\mathrm{CO}_{2} \quad 5-10 \%$ and subcultured to blood agar every $24 \mathrm{~h}$. If a sample is likely to be heavily contaminated, such as from soil, faeces, or animal tissue, selective measures are required [2]. A number of selective and enrichment broths have been described. The most commonly used is Erysipelothrix selective broth (ESB), a liquid medium containing serum, tryptose, neomycin, vancomycin and kanamycin [88]. Packer's medium (SACV) makes use of the organism's tolerance of sodium azide and crystal violet [29], and is frequently used for subculture after growth in ESB [18]. Modified blood azide (MBA) is similar to SACV, but does not include crystal violet [89]. Bohm's medium contains azide, kanamycin, phenol and water blue [8].

Incubation of liquid media at $37^{\circ} \mathrm{C}$ for $18-24 \mathrm{~h}$ is usually sufficient for growth. Colonies are visible after $24 \mathrm{~h}$ on most solid media, although incubation for $48 \mathrm{~h}$ is recommended for ESB and $72 \mathrm{~h}$ for SACV. An alternative for biopsy specimens is to refrigerate them at $4-5^{\circ} \mathrm{C}$ for $4-5$ weeks in a liquid enrichment media and then subculture to SACV [15].

Each medium has advantages, but none is ideal. ESB is still regarded as the best selective medium, despite a report showing that some strains grow poorly due to kanamycin susceptibility [90]. MBA requires less incubation time than SACV, but is not as selective and is not suitable for heavily contaminated samples [89]. Bohm's medium does not seem to have been widely used, although the reasons for this are unclear.

\section{Mouse protection test}

This test is traditionally regarded as the best confirmatory test of $E$. rhusiopathiae identity, because most strains of the organism are highly virulent for laboratory mice. One group of animals is inoculated subcutaneously with a 24-h broth culture and equine hyperimmune $E$. rhusiopathiae antiserum, and the second group receives broth culture but not antiserum. If the organism is E. rhusiopathiae the second group, but not the first, will die within 5-6 days [2]. However, strains must be pathogenic for mice for this method to be useful. While this test is useful in studies of swine erysipelas, the reliability of this method for human pathogenic strains remains unknown.

\section{Fluorescent antibody test}

Direct and indirect assays have been used to confirm the identity of E. rhusiopathiae in tissues [91, 92], in broth [93] and in human infection [94]. However, Harrington [93] noted that this method was not as sensitive as cultural methods, and as a result it has not been used widely.

\section{API Coryne system}

The API Coryne system (bioMérieux) is a commercial strip system for the identification of coryneform bacteria. Soto [28] compared conventional biochemical reactions with the commercial system for corynebacteria and related genera, including Erysipelothrix. The system had few misidentifications and all four strains of $E$. rhusiopathiae tested were correctly identified by use of the strip. The investigators concluded that the commercial strip was a good alternative to traditional biochemical methods, permitting reliable and rapid identification of coryneform bacteria.

\section{PCR}

Two PCR methods are available for the detection of Erysipelothrix species [45,95]. While they were both developed for swine erysipelas, a PCR method was employed for detection of organisms in human specimens in a recent study in Australia [78]. Makino et al. [95] based their primers on a region of the $16 \mathrm{~S}$ rRNA gene, specific to Erysipelothrix but shared by both $E$. rhusiopathiae and E. tonsillarum. Shimoji et al. [45] made use of the avirulent transposon mutant created during capsule studies to develop an E. rhusiopathiaespecific PCR. The primers were designed from sequences presumed to be associated with the virulence of E. rhusiopathiae. This assay would be particularly useful for monitoring of swine disease. However, the value of this test in a human clinical situation is uncertain, due to the lack of information regarding the pathogenicity of E. tonsillarum for humans. The time saved by using the PCR is the greatest advantage it has over all other methods for detecting Erysipelothrix.

Erysipelothrix PCR is very sensitive; Makino et al. [95] detected $<20$ bacteria in a mouse spleen, although the limit detected by Shimoji [45] was only 1000 per reaction mixture, despite a broth enrichment procedure and the use of a DNA extraction kit. Other advantages include the ability to detect an organism in a contaminated sample, as the primers recognise only the specific sequences. With the Makino PCR, the organism does not have to be alive for detection. However, a major drawback is that organism viability cannot be assessed and other procedures would be required for this task. The use of broth enrichment as described by Shimoji [45] could be used to overcome this problem; a modification of this technique was also used in the Australian study [78]. 
PCR is a powerful tool for the detection of Erysipelothrix in all kinds of samples and is able to overcome many problems inherent in other diagnostic methods. However, it is likely that a combination of culture and molecular techniques will be used for accurate diagnosis in the future.

\section{Conclusions}

Although uncommon, it is likely that human infections with E. rhusiopathiae are under-diagnosed. The organism's slow growth and small colony size mean that it may be overlooked in the routine diagnostic laboratory or overgrown with secondary pathogens such as Staphylococcus aureus and Streptococcus pyogenes. A high index of suspicion and the application of modern molecular techniques will no doubt improve this situation. As a pathogen of animals, particularly swine, Erysipelothrix is of great economic importance and good animal husbandry practice is essential to reduce impact. However, the organism's resilience and ability to survive are important in both human and veterinary medicine. Most human infections result from occupational exposure and this possibility can be reduced through awareness and safe work practices.

\section{References}

1. Reboli AC, Farrar WE. Erysipelothrix rhusiopathiae: an occupational pathogen. Clin Microbiol Rev 1989; 2: 354-359.

2. Jones D. Genus Erysipelothrix Rosenbach1909367AL. In: Sneath PHA, Mair NS, Sharpe ME, Holt JG (eds) Bergey's Manual of systematic bacteriology, 9th edn, vol 2. Baltimore, Williams and Wilkins. 1986: 1245-1249.

3. Reboli AC, Farrar WE. The genus Erysipelothrix. In: Balows A, Truper HG, Dworkin M, Harder W, Schleifer K (eds) The prokaryotes. A handbook on the biology of bacteria: ecophysiology, isolation, identification, applications. New York, Springer-Verlag. 1992: 1629-1642.

4. Takahashi T, Fujisawa $\mathrm{T}$, Benno $\mathrm{Y}$ et al. Erysipelothrix tonsillarum sp. nov. isolated from tonsils of apparently healthy pigs. Int J Syst Bacteriol 1987; 37: 166-168.

5. Woodbine M. Erysipelothrix rhusiopathae: bacteriology and chemotherapy. Bacteriol Rev 1950; 14: 161-178.

6. Wood RL. Erysipelothrix infection. In: Hubbert WT, McCulloch WF, Schnurrenberger PR (eds) Diseases transmitted from animals to man, 6th edn. Springfield, IL, Thomas. 1975: $271-281$.

7. Barber M. Discussion on swine erysipelas infection (Erysipelothrix rhusiopathiae) in man and animals. Proc $R$ Soc Med 1948; 41: 328-330.

8. Ewald FW. The genus Erysipelothrix. In: Starr MP, Stolp H, Trüper HG, Balows A, Schlegel HG (eds) The prokaryotes: a handbook on habitats, isolation, and identification of bacteria, Vol 2. New York, Springer-Verlag. 1981: 1688-1700.

9. Takahashi T, Sawada T, Muramatsu $M$ et al. Serotype, antimicrobial susceptibility, and pathogenicity of Erysipelothrix rhusiopathiae isolates from tonsils of apparently healthy slaughter pigs. J Clin Microbiol 1987; 25: 536-539.

10. Takahashi T, Fujisawa $\mathrm{T}$, Tamura $\mathrm{Y}$ et al. DNA relatedness among Erysipelothrix rhusiopathiae strains representing all twenty-three serovars and Erysipelothrix tonsillarum. Int J Syst Bacteriol 1992; 42: 469-473.

11. Chooromoney KN, Hampson DJ, Eamens GJ, Turner MJ Analysis of Erysipelothrix rhusiopathiae and Erysipelothrix tonsillarum by multilocus enzyme electrophoresis. $J$ Clin Microbiol 1994; 32: 371-376.

12. Takahashi T, Tamura Y, Yoshimura $\mathrm{H}$ et al. Erysipelothrix tonsillarum isolated from dogs with endocarditis in Belgium Res Vet Sci 1993; 54: 264-265.

13. Takahashi T, Tamura Y, Endoh YS, Hara N. Cellular fatty acid composition of Erysipelothrix rhusiopathiae and Erysipelothrix tonsillarum. J Vet Med Sci 1994; 56: 385-387.

14. Grieco MH, Sheldon C. Erysipelothrix rhusiopathiae. Ann NY Acad Sci 1970; 174: 523-532.

15. Cottral GE. Erysipelothrix In: Manual of standardized methods for veterinary microbiology. Ithica, NY, Cornell University Press. 1978: 429-436, 671, 672, 679, 687.

16. García-Restoy E, Espejo E, Bella F, Llebot J. Bacteremia due to Erysipelothrix rhusiopathiae in immunocompromised hosts without endocarditis. Rev Infect Dis 1991; 13: 1252-1253.

17. Shimoji Y, Yokomizo Y, Sekizaki T, Mori Y, Kubo M. Presence of a capsule in Erysipelothrix rhusiopathiae and its relationship to virulence for mice. Infect Immun 1994; 62: 2806-2810.

18. Wood RL. Erysipelas. In: Leman AD, Straw BE, Mengeling WL, D'Allaire S, Taylor DJ (eds) Diseases of swine, 7th edn. Ames, IA, Iowa State University Press. 1992: 475-486.

19. Conklin RH, Steele JH. Erysipelothrix infections. In: Steele JH (ed) CRC handbook. Series in zoonoses, vol 1 (section A). Boca Raton, FL, CRC Press. 1979: 327-337.

20. Gorby GL, Peacock JE. Erysipelothrix rhusiopathiae endocarditis: microbiologic, epidemiologic, and clinical features of an occupational disease. Rev Infect Dis 1988; 10: 317-325.

21. Taylor DJ. Pig diseases, 5th edn Cambridge, Burlington Press. 1990.

22. Shuman RD. Erysipelothrix. In: Davis JW, Anderson RC, Karstad LH, Trainer DO (eds) Infectious and parasitic diseases of wild birds. Ames, IA, Iowa State University Press. 1971: $141-145$.

23. Sneath PHA, Abbott JD, Cunliffe AC. The bacteriology of erysipeloid. $B M J 1951$; 2: 1063-1066.

24. Hunter SH. Some growth requirements of Erysipelothrix and Listeria. J Bacteriol 1942; 43: 629-640.

25. Groschup MH, Timoney JF. Modified Feist broth as a serumfree alternative for enhanced production of protective antigen of Erysipelothrix rhusiopathiae. J Clin Microbiol 1990; 28: 2573-2575.

26. White TG, Shuman RD. Fermentation reactions of Erysipelothrix rhusiopathiae. J Bacteriol 1961; 82: 595-599.

27. Vickers CL, Bierer BW. Triple sugar iron agar as an aid in the diagnosis of erysipelas. $J \mathrm{Am}$ Med Vet Assoc 1958; 133: $543-544$.

28. Soto A, Zapardiel J, Soriano F. Evaluation of API Coryne system for identifying coryneform bacteria. $J$ Clin Pathol 1994; 47: 756-759.

29. Packer RA. The use of sodium azide $\left(\mathrm{NaN}_{3}\right)$ and crystal violet in a selective medium for streptococci and Erysipelothrix rhusiopathiae. J Bacteriol 1943; 46: 343-349.

30. Watts PS. Studies on Erysipelothrix rhusiopathiae. J Pathol Bacteriol 1940; 50: 355-369.

31. Kucsera G. Proposal for standardization of the designations used for serotypes of Erysipelothrix rhusiopathiae (Migula) Buchanan. Int J Syst Bacteriol 1973; 23: 184-188.

32. Kalf GF, White TG. The antigenic components of Erysipelothrix rhusiopathiae. II. Purification and chemical characterization of a type-specific antigen. Arch Biochem Biophys 1963; 102: $39-47$.

33. Norrung V, Molin G. A new serotype of Erysipelothrix rhusiopathiae isolated from pig slurry. Acta Vet Hung 1991; 39: $137-138$.

34. Kucsera G. Serological typing of Erysipelothrix rhusiopathiae strains and the epizootiological significance of the typing. Acto Vet Acad Sci Hung 1979; 27: 19-28.

35. Wood RL, Harrington R. Serotypes of Erysipelothrix rhusiopathiae isolated from swine and from soil and manure of swine pens in the United States. Am J Vet Res 1978; 39: 1833-1840.

36. Tamura Y, Takahashi T, Zarkasie K, Nakamura M, Yoshimura H. Differentiation of Erysipelothrix rhusiopathiae and Erysipelothrix tonsillarum by sodium dodecyl sulfate-polyacrylamide gel electrophoresis of cell proteins. Int $J$ Syst Bacteriol 1993; 43: $111-114$.

37. Ahrne S, Sternstrom I, Jensen NE, Pettersson B, Uhlen $M$, Molin G. Classification of Erysipelothrix strains on the basis of restriction fragment length polymorphisms. Int $J$ Syst Bacteriol 1995; 45: 382-385. 
38. Eamens GJ, Turner MJ, Catt RE. Serotypes of Erysipelothrix rhusiopathiae in Australian pigs, small ruminants, poultry, and captive wild birds and animals. Aust Vet $J$ 1988; 65: 249-252.

39. Takahashi T, Sawada T, Seto K, Muramatsu M, Maruyama T, Kanzaki M. Pathogenicity of Erysipelothrix rhusiopathiae strains of serovars $1 \mathrm{a}, 3,5,6,8,11,21$, and type $\mathrm{N}$, isolated from slaughter pigs affected with chronic erysipelas. Nippon Juigaku Zasshi 1985; 47: 1-8.

40. Norrung V. Studies on Erysipelothrix insidiosa - S. rhusiopathiae. I. Morphology, cultural features, biochemical reactions and virulence. Acta Vet Scand 1970; 11: 577-585.

41. Krasemann C, Muller HE. [The virulence of Erysipelothrix rhusiopathiae strains and their neuraminidase production.] Zentralbl Bakteriol Orig A 1975; 231: 206-213.

42. Takahashi T, Hirayama N, Sawada T, Tamura Y, Muramatsu M. Correlation between adherence of Erysipelothrix rhusiopathiae strains of serovar la to tissue culture cells originated from porcine kidney and their pathogenicity in mice and swine. Vet Microbiol 1987; 13: 57-64.

43. Franz B, Davies ME, Horner A. Localization of viable bacteria and bacterial antigens in arthritic joints of Erysipelothrix rhusiopathiae-infected pigs. FEMS Immunol Med Microbiol 1995; 12: $137-142$

44. Shimoji Y, Yokomizo Y, Mori Y. Intracellular survival and replication of Erysipelothrix rhusiopathiae within murine macrophages: failure of induction of the oxidative burst of macrophages. Infect Immun 1996; 64: 1789-1793.

45. Shimoji Y, Mori Y, Hyakutake K, Sekizaki T, Yokomizo Y. Use of an enrichment broth cultivation-PCR combination assay for rapid diagnosis of swine erysipelas. J Clin Microbiol 1998; 36: $86-89$.

46. Shimoji Y, Mori Y, Sekizaki T, Shibahara T, Yokomizo Y. Construction and vaccine potential of acapsular mutants of Erysipelothrix rhusiopathiae: use of excision of $\operatorname{Tn} 916$ to inactivate a target gene. Infect Immun 1998; 66: 3250-3254.

47. Griffiths GL, Buller N. Erysipelothrix rhusiopathiae infection in semi-intensively farmed emus. Aust Vet $J$ 1991; 68: $121-122$.

48. Rowsell HC. The effects of stomach contents and the soil on the viability of Erysipelothrix rhusiopathiae. J Am Vet Med Assoc 1958; 132: 357-361.

49. Stephenson EH, Berman DT. Isolation of Erysipelothrix rhusiopathiae from tonsils of apparently normal swine by two methods. Am J Vet Res 1978; 39: 187-188.

50. Wood RL. Isolation of pathogenic Erysipelothrix rhusiopathiae from feces of apparently healthy swine. Am J Vet Res 1974; 35: 41-43.

51. Shuman RD. Erysipelas. In: Davis JW, Karstad LH, Trainer DO (eds) Infectious diseases of wild mammals. Ames, IA, Iowa State University Press. 1970: 267-272.

52. Heilman FR, Herrell WE. Penicillin in the treatment of experimental infections due to Erysipelothrix rhusiopathiae. Proc Staff Meet Mayo Clin 1944; 19: 340-345.

53. Klauder JV. Erysipeloid and swine erysipelas in man. A clinical and bacteriologic review; swine erysipelas in the United States. JAMA 1926; 86: 536-541.

54. Murase N, Suzuki K, Isayama Y, Murata M. Studies on the typing of Erysipelothrix rhusiopathiae. III. Serological behaviour of the strains isolated from the body surface of marine fishes and their epizootiological significance in swine erysipelas. Jpn J Vet Sci 1959; 21: 215-219.

55. Proctor DM, Richardson IM. A report on 235 cases of erysipeloid in Aberdeen. Br J Ind Med 1954; 11: 175-179.

56. Klauder JV. Erysipeloid as an occupational disease. JAMA 1938; 111: $1345-1348$.

57. Weaver RE. Erysipelothrix. In: Lennette A, Balows A, Hausler WJ, Shadomy HJ (eds) Manual of clinical microbiology, 4th edn. Washington, DC, American Society for Microbiology. 1985: 209-210.

58. Wood RL. Survival of Erysipelothrix rhusiopathiae in soil under various environmental conditions. Cornell Vet 1973; 63: $390-410$.

59. Ternstrom A, Molin G. Incidence of potential pathogens on raw pork, beef and chicken in Sweden, with special reference to Erysipelothrix rhusiopathiae. J Food Protection 1987; 50: $141-146$.

60. Stenstrom I-M, Norrung V, Ternstrom A, Molin G. Occurrence of different serotypes of Erysipelothrix rhusiopathiae in retail pork and fish. Acta Vet Scand 1992; 33: 169-173.
61. McGinnes GF, Spindle F. Erysipeloid condition among workers in a bone button factory due to the bacillus of swine erysipelas. Am J Public Health 1934; 24: 32-35.

62. Hunter D. The diseases of occupations, 5th edn. London, English University Press. 1975: 709-712.

63. Hillenbrand FKM. Whale finger and seal finger: their relation to erysipeloid. Lancet $1953 ; 1$ : $680-681$.

64. Connor MP, Green AD. Erysipeloid in a sheep farmer with coexisting orf. $J$ Infect 1995 ; 30: 161-163.

65. McClain JB. Erysipelothrix rhusiopathiae. In: Mandell GL, Douglas RG, Bennett JE (eds) Principles and practice of infectious diseases, 3rd edn. New York, Churchill Livingstone. 1990: 1599-1600.

66. King PF. Erysipeloid. Survey of 115 cases. Lancet 1946; 2: 196-198.

67. Nelson E. 500 cases of erysipeloid. Rocky Mountain Med J 1955; 52: 40-42.

68. Klauder JV. Erysipelothrix rhusiopathiae infection in swine and in human beings: comparative study of the cutaneous lesions. Arch Dematol Syphil 1944; 50: 151-159.

69. Ehrlich JC. Erysipelothrix rhusiopathiae infection in man. Report of a case with cutaneous bullae, in which cure was achieved with penicillin. Arch Intern Med 1946; 78: 565-577.

70. Fakoya A, Bendall RP, Churchill DR, Doherty JF, Ridgway GL. Erysipelothrix rhusiopathiae bacteraemia in a patient without endocarditis. $J$ Infect 1995; 30: 180-181.

71. Torkildsen A. Intracranial erysipeloid (swine erysipelas) abscess. Variety of abscess not hitherto observed. Bull Hyg 1943: 1013.

72. Silberstein EB. Erysipelothrix endocarditis: report of a case with cerebral manifestations. JAMA 1965; 191: 862-864.

73. Schuster MG, Brennan PJ, Edelstein P. Persistent bacteremia with Erysipelothrix rhusiopathiae in hospitalized patient. Clin Infect Dis 1993; 17: 783-784.

74. Hollick GE, Edinger RE. Erysipelothrix bacteremia. Clin Microbiol Newsl 1995; 17: 9-16.

75. Giménez M, Fernández P, Padilla E, Matas L, Ausina V. Endocarditis and acute renal failure due to Erysipelothrix rhusiopathiae. Eur J Clin Microbiol Infect Dis 1996; 15: 347-348.

76. Totemchokchyakarn K, Janwityanujit S, Sathapatayavongs B, Puavilai S. Erysipelothrix rhusiopathiae septicemia in systemic lupus erythematosus. Int $J$ Dermatol 1996; 35: 818-820.

77. Jones N, Khoosal M. Erysipelothrix rhusiopathiae septicemia in a neonate. Clin Infect Dis 1997; 24: 511.

78. Brooke CJ, McLaughlin V, Mee BJ, Riley TV. An investigation of 'crayfish poisoning' in Western Australia. Med J Aust 1999; 170: 288 .

79. Sheard K, Dicks HG, Skin lesions among fishermen at Houtman's Abrolhos, Western Australia, with an account of erysipeloid of Rosenbach. Med J Aust 1949; 2: 352-354.

80. Takahashi T, Sawada T, Ohmae $\mathrm{K}$ et al. Antibiotic resistance of Erysipelothrix rhusiopathiae isolated from pigs with chronic swine erysipelas. Antimicrobial Agents Chemother 1984; 25: $385-386$.

81. Venditti M, Gelfusa V, Tarasi A, Brandimarte C, Serra P. Antimicrobial susceptibilities of Erysipelothrix rhusiopathiae. Antimicrob Agents Chemother 1990; 34: 2038-2040.

82. Gledhill AW. Discussion on swine erysipelas infection (Erysipelothrix rhusiopathiae) in man and animals. Proc $R$ Soc Med 1948; 41: 330-332.

83. Noguchi N, Sasatsu M, Takahashi T, Ohmae K, Terakado N, Kono M. Detection of plasmid DNA in Erysipelothrix rhusiopathiae isolated from pigs with chronic swine erysipelas. $J$ Vet Med Sci 1993; 55: 349-350.

84. McCarty D, Bornstein S. Erysipelothrix endocarditis. Report on a septicemic form of the erysipeloid of Rosenbach. Am J Clin Pathol 1960; 33: 39-42.

85. Spencer R. The sanitation of fish boxes. I. The quantitative and qualitative bacteriology of commercial wooden fish boxes. $J$ Appl Bacteriol 1959; 22: 73-84.

86. Mutalib AA, King JM, McDonough PL. Erysipelas in caged laying chickens and suspected erysipeloid in animal caretakers. $J$ Vet Diagn Invest 1993; 5: 198-201.

87. Price JEL, Bennett WEJ. The erysipeloid of Rosenbach. $B M J$ 1951; 2: 1060-1062.

88. Wood RL. A selective liquid medium utilizing antibiotics for isolation of Erysipelothrix insidiosa. Am $J$ Vet Res 1965; 26: $1303-1308$. 
89. Harrington R, Hulse DC. Comparison of two plating media for the isolation of Erysipelothrix rhusiopathiae from enrichment broth culture. Appl Microbiol 1971; 22: 141-142.

90. Bratberg AM. Observations on the utilization of a selective medium for the isolation of Erysipelothrix rhusiopathiae. Acta Vet Scand 1981; 22: 55-59.

91. Dacres WG, Groth AH. Identification of Erysipelothrix insidiosa with fluorescent antibody. $J$ Bacteriol 1959; 78: 298-299.

92. Avilag C, Unzucta BB, Olguin RF. Indirect immunofluorescence test for the diagnosis of porcine erysipelas. Veterinaria
(Mexico) 1972; 3: 33-39.

93. Harrington R, Wood RL, Hulse DC. Comparison of a fluorescent antibody technique and cultural method for the detection of Erysipelothrix rhusiopathiae in primary broth cultures. Am J Vet Res 1974; 35: 461-462.

94. Heggers JP, Buddington RS, McAllister HA. Erysipelothrix endocarditis diagnosis by fluorescent microscopy: report of a case. Am J Clin Pathol 1974; 62: 803-806.

95. Makino S, Okada Y, Maruyama $\mathrm{T}$ et al. Direct and rapid detection of Erysipelothrix rhusiopathiae DNA in animals by PCR. J Clin Microbiol 1994; 32: 1526-1531. 